\title{
Stability Of Cosmological Models
}

\author{
M.I.Wanas ${ }^{1}$ andM.A.Bakry ${ }^{2}$ \\ (1. Astronomy and Meteorology Department, Faculty of Science,Cairo \\ University, , Giza, Egypt. E.mail wanas@frcu.eun.eg) \\ (2. Department of Mathematics, Faculty of Education, Ain Shams \\ University, Cairo, Egypt.)
}

\begin{abstract}
The stability problem in cosmology is studied using the equations of geodesic deviation. General conditions for stability are obtained. The method is applied to a number of cosmological models, resulting from different field theories. The results are compared with those obtained from FRW -models.
\end{abstract}

Most of the cosmological models assume the validity of the cosmological principle. So, if the universe is isotropic and homogeneous, then what is the origine of large scale structures in the universe?. These structures cannot be formed in stable cosmological models. Therefore, it is of importance to study the stability of models as a first step to answer the previous question.

Generalizing the classical scheme, for studying the stability of a dynamical system, the authors found that stability can be studied [1] by examining the limit $\left(x^{1}=r, x^{2}=\theta, x^{3}=\phi, x^{4}=t\right)$

$$
\lim _{t \rightarrow b} \xi^{\alpha}
$$

where $[a, b]$ is the interval in which the functions $\xi^{\alpha}$ behave monotonically. The functions $\xi^{\alpha}$ are the components of the deviation vector resulting as a solution of the geodesic deviation equation [2],

$$
\frac{d^{2} \xi^{\alpha}}{d s^{2}}+2\left\{\begin{array}{l}
\alpha \\
\beta \gamma
\end{array}\right\} U^{\beta} \frac{d \xi^{\gamma}}{d s}+\left\{\begin{array}{l}
\alpha \\
\beta \gamma
\end{array}\right\}, \lambda U^{\beta} U^{\gamma} \xi^{\lambda}=0,
$$


where $\left\{\begin{array}{l}\alpha \\ \beta \gamma\end{array}\right\}$ is Christoffel symbol, $U^{\beta}$ is the unit vector resulting from the solution of the geodesic equation:

$$
\frac{d U^{\alpha}}{d s}+\left\{\begin{array}{l}
\alpha \\
\beta \gamma
\end{array}\right\} U^{\beta} U^{\gamma}=0 .
$$

If the limit (1) tends to infinity as $t$ tends to $b$ then the system will be unstable, otherwise the system will be stable. The following scheme is suggested to study the stability of cosmological models, assuming the validity of the cosmological principle. This scheme can be applied as follows: 1- Using the values of Christoffel symboles, of a certain model, to solve (3) we get $U^{\alpha}$. 2- Using $\left\{\begin{array}{l}\alpha \\ \beta \gamma\end{array}\right\}$ and $U^{\beta}$ to solve (2) we get $\xi^{\alpha}$. 3Examining the limit (1) to see whether the model is stable or not.

The study of stability of cosmological models resulting from the following different field theories: 1- the General Theory of Relativity, 2the Relativistic Theory of Gravitation [3], 3- the Generalized Field Theory [4], 4 Moller's Tetrad Theory of Gravitation [5], shows [1] that the suggested scheme represents an easy and fast method for exploring the stability of any cosmological model.

\section{References}

[1]M.I.Wanas and M.A.Bakry (1994) Astrophys. Space Sci. (to appear). [2]S. Weinberg (1972) "Gravitation and Cosmology", John Wiley Sons.

[3]A.A.Logonov and M.A.Mestvirishvilli (1989) "The Relativistic Theory of Gravitation" Mir Pub.

[4]F.I.Mikhail and M.I.Wanas (1977) Proc. Roy. Soc. Lond.A, 356, 471.

[5]C.Moller (1978) Mat. Fys. Skr. Dan. Vid. Selsk 39, 13, 1. 